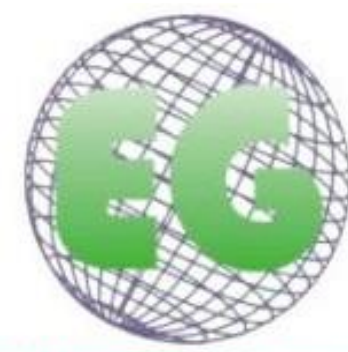

ISSN 1695-6141 $N^{\circ} 53$

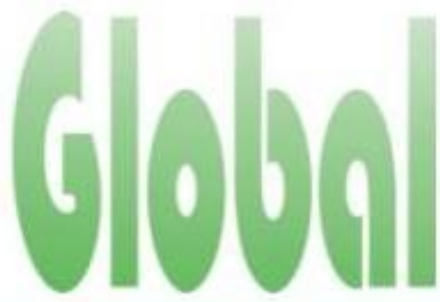

\title{
Infección relacionada con la Asistencia a la Salud en Unidad de
} Cuidados Intensivos Adulto

Infecção relacionada à Assistência à Saúde em Unidade de Terapia Intensiva Adulto Infection related to Health Care in an adult Intensive Care Unit

Luiz Antônio Bergamim Hespanhol ${ }^{1}$

Semírames Cartonilho de Souza Ramos²

Orácio Carvalho Ribeiro Junior ${ }^{3}$

Tatiane Silva de Araújo ${ }^{4}$

Aline Batista Martins

${ }^{1}$ Enfermero Residente en Unidad de Cuidados Intensivos de la Universidad Federal do Amazonas/Hospital Universitario Getúlio Vargas.Brasil. bergamimuepa@hotmail.com.

${ }^{2}$ Profesora Doctora de la Escuela de Enfermería de Manaus de la Universidad Federal do Amazonas.Brasil.

${ }^{3}$ Enfermero. Alumno de Máster del Instituto Leônidas e Maria Deane/ILMD/Fundación Osvaldo Cruz. Brasil.

${ }^{4}$ Residente en Enfermería Obstétrica de la Universidad Federal do Amazonas. Brasil.

\section{http://dx.doi.org/10.6018/eglobal.18.1.296481}

Recibido: 8/06/2017

Aceptado: 9/11/2017

\section{RESUMEN:}

El presente estudio tiene como objetivo caracterizar los casos de infecciones relacionadas a la asistencia a la salud ocurridas en una $\mathrm{UCl}$ adulto. Estudio del tipo descriptivo, exploratorio, documental y con abordaje cuantitativo. La muestra fue constituida por archivos y fichas de control de infección hospitalaria de la $\mathrm{CCIH}$ de todos los casos de infecciones ocurridas en la $\mathrm{UCl}$ del Hospital Universitario Getúlio Vargas en Manaus - Amazonas en el periodo de enero del 2013 a diciembre del 2014. Fueron seleccionados 75 pacientes, siendo la mayoría pertenecientes al sexo femenino $(60 \%)$, con predominio de ancianos (36,5\%). Fueron clasificados como quirúrgicos $(45,3 \%)$ y tuvieron permanencia en la UCI de 16 o más días (42,7\%). En cuanto al perfil de las infecciones, hubo predominio de las relacionadas al tracto respiratorio $(46,2 \%)$ y corriente sanguínea $(26,6 \%)$, llamando atención para la Neumonía Asociada a la Ventilación Mecánica $(35,2 \%)$. El estudio mostró que hay predominio del diagnóstico clínico, de laboratorio y de imagen en la identificación de las IRAS, sumando (62,4\%), descuidando la realización de cultivos (37,5\%). Entre los microorganismos identificados existe el predominio de los gram negativos $(28,1 \%)$. La mayor ocurrencia de infección por dispositivos invasivos ocurrió en el uso del tubo orotraqueal (48\%). Hubo correlación fuerte y significativa entre el resultado muerto y la cantidad de infecciones presentes $(p=0,02)$ y cuanto al número de patógenos aislados en cada paciente $(p=0,03)$. Se vuelve imprescindible la correcta investigación de los casos de IRAS, atendiendo a los diferentes factores asociados a su ocurrencia, resultando evidente la necesidad de mayor vigilancia epidemiológica de las infecciones en la Unidad de Cuidadps Intensivos.

Palabras clave: Unidad de Cuidados Intensivos; Infección Relacionada a la Asistencia a la Salud; Dispositivos Invasivos. 


\section{RESUMO:}

O presente estudo tem como objetivo caracterizar os casos de infecções relacionadas à assistência à saúde ocorridas em uma UTI adulto. Estudo do tipo descritivo, exploratório, documental e com abordagem quantitativa. A amostra foi constituída por prontuários e fichas de controle de infecção hospitalar da CCIH de todos os casos de infecções ocorridas na UTI do Hospital Universitário Getúlio Vargas em Manaus - Amazonas no período de janeiro de 2013 a dezembro de 2014. Foram selecionados 75 pacientes, sendo a maioria pertencentes ao sexo feminino $(60 \%)$, com predomínio de idosos (36,5\%). Foram classificados como cirúrgicos $(45,3 \%)$ e tiveram permanência na UTI de 16 ou mais dias $(42,7 \%)$. Quanto ao perfil das infecções, houve predomínio das relacionadas ao trato respiratório $(46,2 \%)$ e corrente sanguínea $(26,6 \%)$, chamando atenção para a Pneumonia Associada à Ventilação Mecânica (35,2\%). O estudo mostrou ainda que há predomínio do diagnóstico clínico, laboratorial e de imagem na identificação das IRAS, somando $(62,4 \%)$, negligenciando a realização de culturas $(37,5 \%)$. Dentre os microorganismos identificados existe o predomínio dos gram-negativos $(28,1 \%)$. A maior ocorrência de infecção por dispositivos invasivos ocorreu no uso do tubo orotraqueal (48\%). Houve correlação forte e significativa entre o desfecho óbito e a quantidade de infecções presentes $(p=0,02)$ e quanto ao número de patógenos isolados em cada paciente $(p=0,03)$. Torna-se imprescindível a correta investigação dos casos de IRAS, tendo em vista os diferentes fatores associados a sua ocorrência, ficando evidente a necessidade de maior vigilância epidemiológica das infecções em Unidade de Terapia Intensiva.

Palavras chave: Unidade de Terapia Intensiva; Infecção Relacionada à Assistência à Saúde; Dispositivos Invasivos.

\section{ABSTRACT:}

The present study aims to characterize the cases of Infections related to health care Occurred in an adult ICU. A descriptive, exploratory, documental and quantitative tipe. The sample consisted of medical records and files Of infection control of the $\mathrm{CCIH}$ of all cases of infections occurred in the ICU of Hospital Universitário Getúlio Vargas in Manaus - Amazonas from January 2013 to December 2014. Seventy-five patients were selected, Being the majority belonging to the female sex $(60 \%)$, With predominance of the elderly (36.5\%). They were classified as surgical $(45.3 \%)$ and had ICU stay of 16 or more days $(42.7 \%)$. Regarding the profile of infections, there were predominant respiratory tract infections (46.2\%) and blood flow (26.6\%), calling attention to associated pneumonia with Mechanical Ventilation (35.2\%). The study also showed that there is a predominance of clinical, laboratory and imaging diagnosis in the identification of IRAS, adding up to $62.4 \%$, neglecting to perform cultures $37.5 \%$. Among the identified microorganisms there is a predominance of gram-negative $28.1 \%$. The highest occurrence of infection by invasive devices occurred in the use of the orotracheal tube $48 \%$. There was a strong and significant correlation between the death outcome and the amount of infections present $(p=0,02)$ and the number of pathogens isolated in each patient $(p=0,03)$. The correct investigation of IRAS cases is essential, considering the different factors associated with its occurrence, evidencing the need for greater epidemiological surveillance of infections in the Intensive Care Unit.

Keywords: Intensive Care Unit; Health Care Related Infection; Invasive Devices.

\section{INTRODUCCIÓN}

El entendimiento del término Infecciones Hospitalarias (IH) viene siendo sustituido en los últimos años por el Ministerio de la Salud y Agencia Nacional de Vigilancia Sanitaria por el término Infección Relacionada a la Asistencia a la Salud (IRAS) tanto en la perspectiva de la prevención cuanto en el control de las infecciones, dado que estas pasan a ser consideradas como evento que puede ocurrir no sólo en el ámbito hospitalario, sino en todos los ambientes que prestan asistencia a la salud ${ }^{(1)}$.

Las IRAS, por la longevidad que se presentan pueden ser consideradas como una de las lesiones más antiguas y que generan preocupación para la humanidad, visto que amenazan la calidad y la evolución de los servicios de atención a la salud, principalmente en los hospitales. La Organización Mundial de la Salud (OMS) reconoce que las IRAS son un problema de Salud Pública y que son necesarias acciones estratégicas con vistas a la reducción o eliminación de este problema, 
acciones estas que deben ser desarrolladas en todas las esferas gubernamentales y de forma articulada ${ }^{(2)}$.

En el ambiente hospitalario, más específicamente, las IRAS son las infecciones que suceden después de la admisión en el hospital y que pueden desarrollarse durante la internación o después del alta, desde que tengan relación con la internación o procedimientos realizados durante la misma ${ }^{(2)}$.

En virtud del ambiente asistencial invasivo de la $\mathrm{UCI}$, necesario en la atención de las condiciones críticas del proceso de enfermedad, los pacientes son más propensos a adquirir infecciones y la ocurrencia de estas acarrea innúmerables problemas tanto en la vertiente del paciente, como en la prolongación de su permanencia en el hospital, demora en su recuperación y agravamiento de la condición clínica existente, cuanto para la institución y el estado, considerando que la incidencia de IRAS es uno de los criterios para la evaluación de la calidad de los servicios de los hospitales, además del aumento de los costos financieros del estado con el sector salud ${ }^{(3)}$.

De esta forma, las IRAS constituyen un serio problema de salud pública que necesita ser solucionado y la vigilancia epidemiológica se constituye como herramienta principal para el conocimiento de aquellas, pues permite la observación activa, continua y sistemática de la aparición y distribución de esas infecciones entre los pacientes institucionalizados, así como los eventos y condiciones que se relacionan con el riesgo de su aparición y comportamiento, con vistas a la ejecución de acciones oportunas para el control ${ }^{(2,3)}$.

A pesar de los notables avances que se observaron en las últimas décadas en el sentido de eliminar o controlar las IRAS, muchos son los desafíos que aún se presentan para la implementación efectiva de las estrategias para el enfrentamiento de esta problemática, entre ellos las lagunas de conocimiento sobre la aparición y la distribución de las IRAS en las diversas regiones del país y las deficiencias en la recopilación de informaciones que permitan el direccionamiento de acciones y control de efectividad de las mismas desde el nivel nacional, hasta las unidades de internación, como las UCls, que son consideradas áreas críticas para esas infecciones $^{(4)}$.

Ante lo expuesto, el presente estudio tiene como objetivo caracterizar los casos de infecciones relacionadas a la asistencia a la salud, ocurridos en la UCI de un Hospital Universitario.

\section{MATERIAL Y MÉTODOS}

El estudio es del tipo descriptivo, exploratorio, documental y con abordaje cuantitativo. La investigación fue realizada en el Hospital Universitario Getúlio Vargas (HUGV), localizado en el municipio de Manaus, estado de Amazonas. El hospital está vinculado a la Universidad Federal de Amazonas (UFAM) y administrado actualmente por la Empresa Brasileña de Servicios Hospitalarios (EBSERH), está situado en la calle Afonso Pena, s/n, barrio Praça 14 de janeiro.

La población de este estudio se refiere a los casos de pacientes internados en la UCI del HUGV que desarrollaron infecciones relacionadas a la asistencia a la salud. La muestra fue constituida de archivos y fichas de control de infección hospitalaria de la 
$\mathrm{CClH}$ de todos los casos de infecciones ocurridas en la $\mathrm{UCI}$ del referido hospital en el periodo de enero del 2013 a diciembre del 2014.

La recopilación de datos se realizó en tres meses, en el periodo de julio a septiembre de 2015. Se utilizó como herramienta de recopilación de datos un instrumento estructurado, elaborado por el investigador responsable, con variables relacionadas a las características epidemiológicas y clínicas de los pacientes a los cuales se refiere este estudio.

Los datos fueron cuantificados, tabulados y analizados estadísticamente, utilizando la planilla Software Microsoft de cálculo del Office Excel, versión 2010 para la construcción de tablas que expresen las variables del estudio. Los datos recopilados fueron sometidos a un análisis descriptivo, utilizando como software $R$, versión 3.2.2.

El modelo de regresión logístico fue aplicado con el objetivo de estudiar el efecto de las variables sexo, franja de edad, dispositivos invasivos, entre otras, con relación al desenlace (alta y óbito) referente a los pacientes que desarrollaron infección en la UCI.

Las hipótesis para la Regresión Logística fueron:

- $\mathrm{H}_{0}$ : Las variables influyeron en el desenlace.

- $\mathrm{H}_{1}$ : Las variables no influyeron en el desenlace.

El p-valor siendo menor que el nivel de significancia de $5 \%$ no se rechaza $\mathrm{H}_{0}$, o sea, las variables influyeron en el desenlace.

Para la ejecución de la investigación se utilizó el Término de Compromiso de Utilización de Datos (TCUD) debido a la imposibilidad de la obtención del Consentimiento Informado de todos los pacientes y por tener que analizarse archivos, correspondiendo al investigador la confidencialidad de los datos obtenidos.

El Término de Dispensa del Término de Consentimiento Libre y Aclarado fue utilizado en razón de la recolección ser realizada sólo por datos secundarios, obtenidos a partir del estudio de archivos con las informaciones referentes a la asistencia prestada a los pacientes.

El proyecto fue sometido al Comité de Ética en Pesquisa (CEP) de la Universidad Federal de Amazonas (UFAM) en Manaus-AM para análisis y posterior parecer.

\section{RESULTADOS}

Fueron admitidos en el año de 2013, en la Unidad de Cuidados Intensivos 277 pacientes, de éstos, 222 recibieron alta de la unidad y 55 vinieron a óbito, de los cuales 51 óbitos ocurrieron en pacientes con IRAS y 19 óbitos relacionados a IRAS. Ya en el año 2014 fueron admitidos 272 pacientes, de éstos, 219 recibieron alta y 53 vinieron a óbito, de los cuales 24 óbitos ocurrieron en pacientes con IRAS y 02 óbitos relacionados a IRAS. La muestra total de casos identificados durante el estudio de pacientes que desarrollaron infecciones relacionadas a dispositivos invasivos fue de 75. 
Tabla 1 - Perfil sociodemográfico y de internación y su relación con el desenlace alta/óbito en pacientes internados en la UCl, Manaus-AM, 2016.

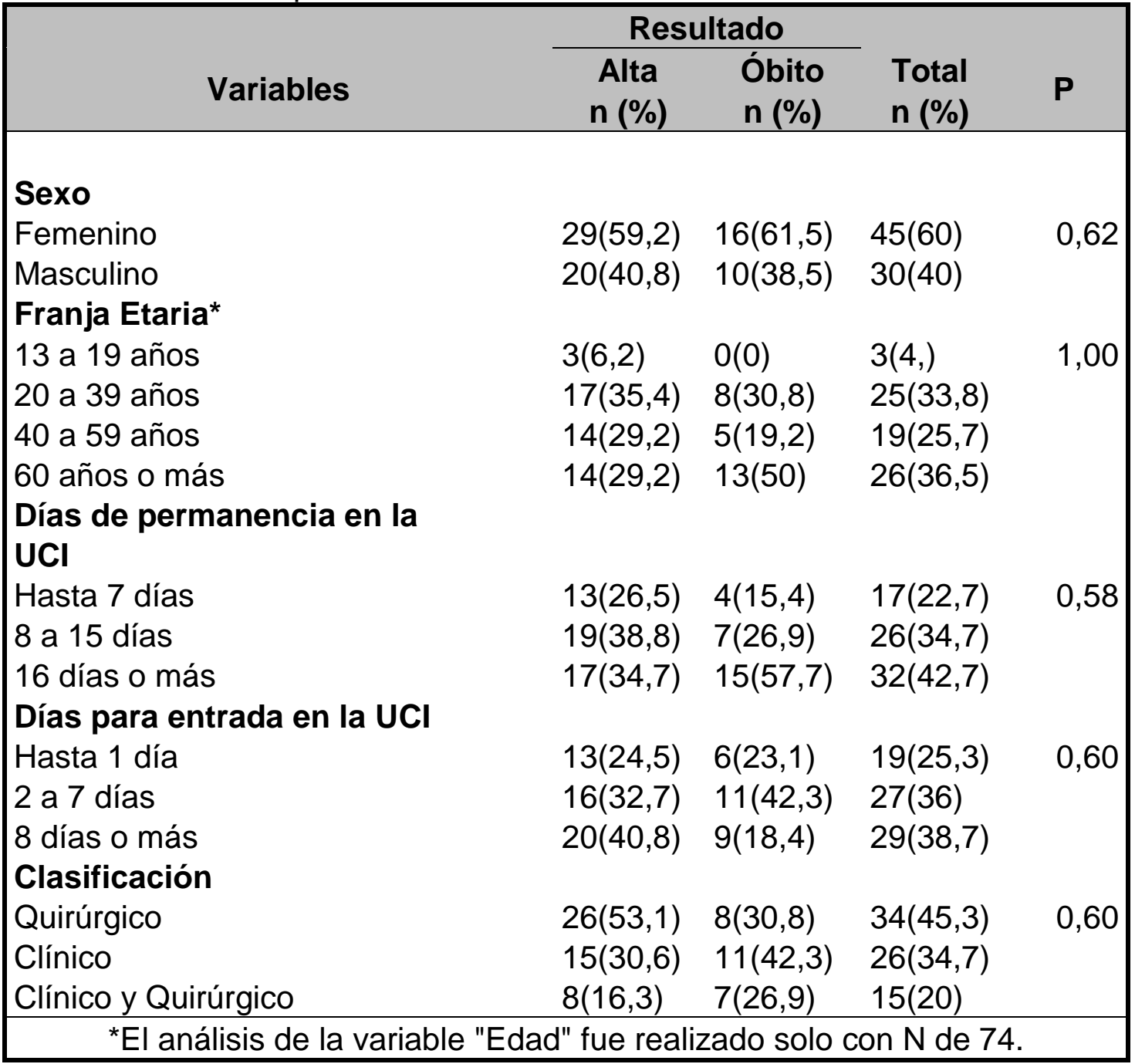

En relación a las características sociodemográficas y de integración, se observa mayor prevalencia de infecciones en los pacientes del sexo femenino $(45 ; 60 \%)$ y ancianos $(27 ; 36,5 \%)$. El mayor porcentaje de los pacientes permanecieron internados en la UCl por más de 15 días $(32 ; 42,7 \%)$ con un promedio y DP de $(26,5 \pm 35,8)$, tiempo mínimo de permanencia de 03 y máximo de 192 días. El tiempo de entrada en la terapia intensiva desde su admisión en cualquier otro sector del hospital fue mayor que 07 días $(29 ; 38,7 \%)$ en su mayoría, con promedio y DP de $(09,4 \pm 19,4)$, tiempo mínimo de 01 y máximo de 60 días. No hubo significancia estadística con la asociación entre las variables presentadas en la tabla 1 con el desenlace alta y óbito $(p>0,05)$. 
Tabla 2: Características clínicas y diagnósticas de las infecciones en pacientes internados en la UCl, Manaus-AM, 2016.

\begin{tabular}{|c|c|}
\hline Variables & $n(\%)$ \\
\hline \multicolumn{2}{|l|}{ Tipo de Infección } \\
\hline Infección Cutánea & $3(2,4)$ \\
\hline Tejidos Blandos (ocular) & $3(2,3)$ \\
\hline Sitio quirúrgico & $4(3,1)$ \\
\hline Sistema Nervioso Central & $5(3,9)$ \\
\hline Tracto Respiratorio (PNM Clínica) & $14(11)$ \\
\hline Tracto Urinario & $20(15,6)$ \\
\hline Infección de Corriente Sanguínea & $34(26,6)$ \\
\hline Tracto Respiratorio (PAV) & $45(35,2)$ \\
\hline \multicolumn{2}{|l|}{ Tipo de Diagnóstico de la Infección } \\
\hline Diag. Clínico & $9(7)$ \\
\hline Diag. Clínico y de Laboratorio & $30(23,4)$ \\
\hline Diag. Clínico y Radiológico & $41(32)$ \\
\hline Cultivos & $48(37,5)$ \\
\hline \multicolumn{2}{|l|}{ Microorganismos aislados } \\
\hline Gram negativa & $36(28,1)$ \\
\hline Gram positiva & $10(7,8)$ \\
\hline Hongos & $2(1,6)$ \\
\hline Sin cultivos & $80(62,5)$ \\
\hline \multicolumn{2}{|l|}{ Resultado } \\
\hline Alta & $49(65,4)$ \\
\hline Óbito & $26(34,6)$ \\
\hline \multicolumn{2}{|c|}{ Tiempo para Confirmación de la Infección } \\
\hline Hasta 7 días & $58(50,9)$ \\
\hline 8 a 15 días & $26(22,8)$ \\
\hline 16 a 30 días & $19(16,7)$ \\
\hline Más de 30 días & $11(9,6)$ \\
\hline
\end{tabular}

La tabla 2 muestra las características clínicas y diagnósticas de los casos de infecciones. La infección que estuvo más presente fue la del tracto respiratorio, del tipo neumonía asociada a la ventilación mecánica (PAV) $(45 ; 35,2 \%)$, con uso del tubo orotraqueal o traqueotomía, como dispositivo de ventilación invasiva pulmonar. La confirmación diagnóstica de las infecciones se dio en su mayoría por medio de criterios clínicos, radiológicos y de laboratorio, esencialmente (80; 62,5\%), con excepción de los diagnósticos realizados por cultivos $48(37,5)$.

Los microorganismos encontrados frecuentemente en los cultivos fueron los gram negativos (36; $28,1 \%)$, entre ellos, Acinetobacter baumannii haemolyticus $(1 ; 0,8 \%)$, Cedecea davisae $(1 ; 0,8 \%)$, Citrobacter diversus $(1 ; 0,8 \%)$, Stenotrophomonas maltophilia $(1 ; 0,8 \%)$, Serratia marcescens $(2 ; 1,6 \%)$, Enterobacter aerogenes (2; $1,6 \%)$, Enterobacter agglomerans (2; 1,6\%), Klebsiella oxytoca (3; 2,3\%), Klebsiella pneumoniae $(6 ; 4,7 \%)$, Escherichia coli $(6 ; 4,7 \%)$, Pseudomonas sp. $(1 ; 0,8 \%)$ e Pseudomonas aeruginosa (10; 7,8\%), las gram positivas (10; 7,8\%) fueron, Enterococcus casseliflavus (1;0,8\%), Streptococcus sp. (1; $0,8 \%)$, Staphylococcus xylosus $(1 ; 0,8 \%)$, Staphylococcus sp. $(1 ; 0,8 \%)$, Staphylococcus coagulase negativa 
$(2 ; 1,6 \%)$ y Staphylococcus aureus $(4 ; 3,1 \%)$. Los hongos presentes en los cultivos fueron representados por Candida sp. (2; 1,6\%).

Tabla 3: Ocurrencia de infección por dispositivos invasivos y su relación con el desenlace alta/óbito en pacientes internados en la UCI, Manaus-AM, 2016.

\begin{tabular}{|c|c|c|c|c|}
\hline \multirow{2}{*}{ Dispositivos } & \multicolumn{2}{|c|}{ Resultado } & \multirow[b]{2}{*}{$\begin{array}{l}\text { Total } \\
\text { n (\%) }\end{array}$} & \multirow[b]{2}{*}{$\mathbf{P}$} \\
\hline & $\begin{array}{l}\text { Alta } \\
\text { n (\%) }\end{array}$ & $\begin{array}{l}\text { Óbito } \\
\text { n (\%) }\end{array}$ & & \\
\hline \multicolumn{5}{|l|}{ Tubo Orotraqueal } \\
\hline 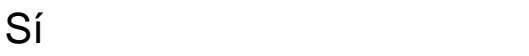 & $23(46,9)$ & $13(50)$ & $36(48)$ & 0,99 \\
\hline No & $26(53,1)$ & $13(50)$ & $39(52)$ & \\
\hline \multicolumn{5}{|l|}{ Traqueotomía } \\
\hline Sí & $8(16,3)$ & $4(15,4)$ & $12(16)$ & 1,00 \\
\hline No & $41(83,7)$ & $22(84,6)$ & $63(84)$ & \\
\hline \multicolumn{5}{|l|}{$\begin{array}{l}\text { Derivación Ventricular } \\
\text { Externa }\end{array}$} \\
\hline Sí & $2(4,1)$ & $2(7,7)$ & $4(5,3)$ & 0,99 \\
\hline No & $47(95,9)$ & $24(92,3)$ & $71(94,7)$ & \\
\hline \multicolumn{5}{|l|}{ Catéter Venoso Periférico } \\
\hline 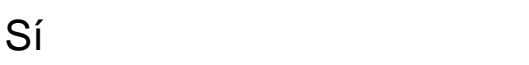 & $3(6,1)$ & $2(7,7)$ & $5(6,7)$ & 1,00 \\
\hline No & $46(93,9)$ & $24(92,3)$ & $70(93,3)$ & \\
\hline \multicolumn{5}{|l|}{ Catéter Venoso Central } \\
\hline No & $42(85,7)$ & $17(65,4)$ & $59(78,7)$ & \\
\hline Sonda Vesical Demora & & & & 1,00 \\
\hline Sí & $10(20,4)$ & $4(15,4)$ & $14(18,7)$ & \\
\hline No & $39(79,6)$ & $22(84,6)$ & $61(81,3)$ & \\
\hline
\end{tabular}

La tabla 3 presenta la ocurrencia de infección en cada dispositivo invasivo durante la asistencia intensiva y su relación con el alta y los óbitos ocurridos en los pacientes. Se llama la atención para el uso del Tubo Orotraqueal, como dispositivo más utilizado dentro de la $\mathrm{UCl}(36 ; 48 \%)$ relacionado a la presencia de IRAS y el Catéter Venoso Central con el mayor número de óbitos $(9 ; 34,6 \%)$ en comparación a las altas $(7 ; 14,3 \%)$. La presencia de infección en cualquiera de los dispositivos presentados no mostró significancia estadística en la asociación con el resultado $(p>0,05)$.

Tabla 4: Infecciones y su relación con el resultado alta/óbito en pacientes internados en la UCl, Manaus-AM, 2016.

\begin{tabular}{|lcccc|}
\hline \multicolumn{1}{|c}{ Variables } & Promedio \pm DP & Mínimo & Máximo & P \\
\hline No de Microorganismos & & & & \\
Aislados & $0,64 \pm 0,98$ & 0 & 5 & 0,02 \\
$N^{\circ}$ de infecciones & $1,70 \pm 1,62$ & 1 & 9 & 0,03 \\
\hline
\end{tabular}

En la tabla 4 se ve la relación estadística entre las variables de infecciones y su resultado, siendo significantes estas asociaciones $(p<0,05)$, o sea cuanto mayor el número de microorganismos aislados y el número de infecciones en un paciente, 
mayor será la probabilidad de óbito en la terapia intensiva, según el modelo de regresión logística aplicado.

\section{DISCUSIÓN}

La UCl es un ambiente caracterizado por recibir pacientes gravemente enfermos y debido a los cuidados altamente invasivos realizados en este ambiente y la propia condición crítica del cliente, son consideradas las unidades hospitalarias en las que se concentran los mayores índices de IH o IRAS, lo que torna a la $\mathrm{UCI}$ un ambiente prioritario para las acciones de prevención y control de infecciones. La alta prevalencia de estas infecciones es un factor que implica muchas veces un resultado desfavorable de estos pacientes en la UCI. Además las IRAS están asociadas a la extensión de internación, mortalidad y morbilidad y altos costos con terapéutica ${ }^{(5)}$.

Con relación al género, este estudio evidenció mayor prevalencia de IRAS en el sexo femenino $(60 \%)$, esta variable cuando comparada a otros estudios muestra la ocurrencia de mayores tasas de IRAS en el sexo masculino, a pesar de esa tendencia ser discreta, no ultrapasando los $60 \%{ }^{(6-10)}$. Estos hallazgos reflejan que hay un predominio de IRAS en el género masculino, mostrándolo como más vulnerable. Sin embargo, este hallazgo puede estar ocurriendo al azar, ya que en ninguno de los estudios supracitados hubo significancia estadística para la variable sexo.

Ya en lo que concierne a la variable edad, los datos de la presente investigación muestran un predominio de IRAS en pacientes con edad superior a los 40 años, hecho evidenciado en otros estudios donde fueron encontrados promedios de edad entre $53 ; 57,3 ; 57$ y 54 respectivamente ${ }^{(6,8-10)}$. Además es posible percibir una considerable tasa $(36,5 \%)$ de pacientes afectados por IRAS con más de 60 años de edad, conforme también encontrado en otros estudios realizados en esta temática ${ }^{(6-7)}$. Muchos factores contribuyen a la ocurrencia de IRAS en todos los ambientes hospitalarios, inclusive aquellos intrínsecos al paciente, siendo la edad avanzada un importante factor ya comprobado en la literatura ${ }^{(11)}$.

En lo que respecta a los días de permanencia en la $\mathrm{UCI}$, se observó que la mayoría de los pacientes (82,4\%) tuvo una estadía en aquel sector superior a 7 días. Resultados semejantes fueron encontrados por estudios desarrollados en otras regiones de Brasil, donde el promedio de internación de los pacientes que desarrollaron IRAS fue de 19,3; 21 y 21,2 días respectivamente ${ }^{(7,10,12)}$. El tiempo de permanencia prolongado en la $\mathrm{UCI}$ se asocia a un mayor riesgo de colonización por patógenos multirresistentes, hecho que favorece la adquisición de IRAS ${ }^{(13)}$.

Está consolidado en la literatura que la permanencia del paciente en el ambiente crítico se configura como un gran factor de riesgo para la adquisición de infecciones, dado que éste sufre gran exposición ambiental en la $\mathrm{UCl}$, lo que aumenta las posibilidades de colonización por microorganismos multirresistentes y también de infección cruzada ${ }^{(14)}$.

Los datos referentes a los días para la entrada en la UCI después de la admisión en el hospital revelan que un porcentaje importante de pacientes $(38,7 \%)$ tuvo un tiempo de hospitalización superior a 8 días, hecho que configura como factor de riesgo aumentado para la precipitación de IRAS por los mismos motivos de larga permanencia en la UTI ${ }^{(13)}$. Además, estos datos son corroborados por un estudio desarrollado en un hospital universitario que detectó un promedio de permanencia de 
36 días entre los pacientes que desarrollaron infección. Este resultado puede estar relacionado con las enfermedades de base de los pacientes que desarrollaron IRAS, dado que el hospital donde se realizó este estudio es de referencia para enfermedades graves y procedimientos quirúrgicos de gran tamaño. Estos acontecimientos de base tienden a desestabilizar los mecanismos de defensa del organismo, causar desnutrición y conferir fallas en las defensas inmunológicas, que aliado al tiempo de permanencia institucional prolongado se vuelven graves factores de riesgo para las IRAS ${ }^{(6,13)}$.

En cuanto a la clasificación del paciente, este estudio demostró una mayor prevalencia de pacientes quirúrgicos $(45,3 \%)$, resultado semejante también encontrado en otro estudio sobre la temática ${ }^{(7)}$. Sin embargo, estos descubrimientos no se revelan padrón en la literatura, pues, estudio más reciente sobre IRAS en UCI mostró descubrimiento inverso de este estudio, donde $83,2 \%$ de la muestra fue clasificada como clínica ${ }^{(8)}$. De esta forma, no es posible establecer una relación exacta sobre la ocurrencia de IRAS y la clasificación del paciente, pues, ambos pueden configurar como factor de riesgo.

Cuando realizada la asociación estadística entre las características sociodemográficas de los pacientes con IRAS con los resultados alta y óbito, no hubo significancia suficiente para relacionar de forma efectiva tales datos con estos 2 desenlaces, conforme observado en la tabla 1.

Al investigar el tipo de infección presente en la unidad se observó una mayor incidencia de infección del tracto respiratorio del tipo neumonía asociada a ventilación mecánica (PAV), seguido de infección de corriente sanguínea y del tracto urinario. Predominancia semejante en relación a topografías de las IRAS en UCI ocurrió en otro estudio ${ }^{(12)}$, que demostró la misma secuencia de acontecimientos en orden decreciente, siendo la prevalencia de infección respiratoria de $48,1 \%$ e ICS $32,1 \%$. Otro estudio también demostró que infecciones respiratorias, de corriente sanguínea y urinarias son las topografías más frecuentes en $\mathrm{UCl}^{(15)}$. Estudio realizado en una $\mathrm{UCI}$ en Rio Grande do Sul identificó que las infecciones respiratorias fueron responsables por $57 \%$ del total en aquella UCI ${ }^{(16)}$. Corroborando con los descubrimientos de este ensayo, el Ministerio de la Salud afirma que infecciones del tracto respiratorio corresponden a aproximadamente $25 \%$ de todas las infecciones adquiridas en las UCIS, estando los pacientes en ventilación mecánica invasiva en un grupo de riesgo aumentado de la neumonía en virtud de tres factores principales: disminución de las defensas del paciente, riesgo elevado de tener las vías aéreas inoculadas con gran cantidad y material contaminado y presencia de microorganismos más agresivos y resistentes a los antimicrobianos en el ambiente, superficies cercanas, materiales y colonizando al propio paciente ${ }^{(17)}$. Es importante resaltar que la neumonía es la principal causa de IRAS en UCl, siendo que su incidencia aumenta en hasta 21 veces en los pacientes sometidos a ventilación mecánica, configurando como principal causa de muerte por infecciones en este contexto ${ }^{(18-19)}$.

Con relación a la forma de diagnóstico de las infecciones, hubo predominio de la clínica manifestada, asociada a los exámenes de laboratorio y radiológicos, y el diagnóstico por cultivo fue realizado en menos de la mitad de los casos. Estos descubrimientos divergen de estudios recientes que caracterizaron las IRAS en UCI donde el diagnóstico por medio de cultivo estuvo presente en tasas que llegaron hasta $90 \%$ de los casos estudiados ${ }^{(10)}$. Al respecto, el Ministerio de la Salud, por medio de sus documentos oficiales, señala que los criterios diagnósticos de las IRAS son 
específicos para cada topografía estudiada, tomando en consideración aspectos clínicos y de laboratorio, sin embargo, debe tener el aislamiento del microorganismo asociado, para una mejor dirección de la terapéutica ${ }^{(17,20)}$.

En lo que concierne a los tipos de microorganismos aislados en las culturas realizadas, se observó predominio de las bacterias gram negativas. Este descubrimiento es corroborado por otros estudios, en los cuales se registraron incidencias de ese grupo de bacterias en $81,1 \%$, $82 \%$ y $72,9 \%$ respectivamente ${ }^{(12,16,21)}$. Infecciones causadas por bacterias gram negativas están ligadas a perfiles de resistencia extremadamente elevados, lo que torna difícil el establecimiento de terapéutica inicial y tiene fuerte impacto en el pronóstico de pacientes críticos, siendo responsables de las altas tasas de mortalidad asociada a las IRAS en $\mathrm{UCl}^{(22)}$.

En lo que se refiere a los resultados de los pacientes con IRAS investigados en este estudio, la mayoría tuvo alta de la unidad (67,4\%). Este descubrimiento es corroborado por un estudio realizado en una UCI de Paraná, donde el resultado alta fue de $61,6 \%{ }^{(23)}$. Otros estudios internacionales traen resultados donde el porcentaje de alta fue de $83,2 \%, 89 \%$ y $87,2 \%$ respectivamente ${ }^{(11,24,25)}$. Estos resultados están directamente asociados a factores relacionados con el cuadro de entrada del paciente en la $\mathrm{UCI}$, al número de dispositivos invasivos instalados y a la presencia de las IRAS en estos pacientes ${ }^{(12)}$.

En cuanto al tiempo para diagnóstico de IRAS después del ingreso en la UCl, este estudio mostró detección precoz en cerca de la mitad de los casos y más de $70 \%$ en hasta 15 días. Estos descubrimientos son semejantes a los encontrados en otro estudio con promedio de tiempo para el diagnóstico de IRAS de 11,7 días ${ }^{(12)}$. Ya en el estudio desarrollado en una UCl de Minas Gerais mostró un tiempo promedio de 5,6 días para el diagnóstico de IRAS, hecho que también corrobora lo presentado en este estudio $^{(8)}$.

En cuanto a la ocurrencia de IRAS de acuerdo con los dispositivos invasivos utilizados, y su relación con el resultado alta y óbito, se nota que tubo orotraqueal, traqueotomía, catéter venoso central y sonda vesical de demora fueron los dispositivos que más estuvieron asociados a la presencia de IRAS, destacando en este estudio el tubo orotraqueal. Estos descubrimientos son semejantes a otros estudios realizados, donde los mismos dispositivos fueron asociados a la presencia de IRAS en UCI, siendo los dispositivos ligados a la ventilación mecánica los responsables por dar sustento al mayor resultado óbito entre los pacientes con $\operatorname{IRAS}^{(10,12)}$.

Es evidente la correlación de uso de dispositivos invasivos y la presencia de IRAS. Estudios ya realizados confirman la relación estadística entre el uso de catéter venoso central y las infecciones de corriente sanguínea y el uso del catéter vesical de demora con las infecciones del tracto urinario ${ }^{(26)}$. Otro estudio demostró que $90 \%$ de los pacientes que desarrollaron neumonía nosocomial en UCI fueron sometidos a intubación traqueal y ventilación mecánica ${ }^{(14)}$.

Este estudio mostró fuerte evidencia, por medio de regresión logística, de la relación estrecha con el resultado óbito y de los aspectos importantes: número de infecciones y número de patógenos asociados. Estos hallazgos sólo refuerzan esa asertiva ya consolidada en muchos estudios realizados en Brasil y en otros países, así como 
aquello que es disertado en la literatura gris, pues estos factores aumentan significativamente la dificultad en el manejo terapéutico y el riesgo de que lo institucionalizado evolucione a un cuadro de sepsis y choque séptico ${ }^{(12,22)}$.

\section{CONCLUSIONES}

Así como es importante la aplicación de nuevas tecnologías sofisticadas en el cuidado al paciente crítico, es fundamental y se torna imperativo el conocimiento de los aspectos clínicos y epidemiológicos de las IRAS en este ambiente de cuidado, visto que la presencia de las mismas hace el servicio extremadamente oneroso y disminuyen la efectividad del papel al cual la UCI se destina.

De esa forma, se puede concluir con este estudio que los pacientes afectados de IRAS en el contexto investigado pertenecía al sexo femenino, con edad igual o superior a los 60 años, en su mayoría fueron clasificados como quirúrgicos y tuvieron una larga permanencia en la $\mathrm{UCl}$. En cuanto al perfil de las infecciones, hubo predominio de las relacionadas al tracto respiratorio, corriente sanguínea y tracto urinario, llamando la atención la Neumonía Asociada a la Ventilación Mecánica (PAV) y su asociación a un mayor resultado óbito entre los pacientes afectados. El estudio mostró además que hay predominio de la clínica asociada a exámenes de laboratorio y de imagen en el diagnóstico de las IRAS y que se descuida la realización de cultivos. Entre los microorganismos identificados existe el predominio de los gram negativos, lo que configura un gran desafío para el correcto y efectivo manejo terapéutico de los pacientes. Los dispositivos más utilizados y que estuvieron asociados a la presencia de IRAS fueron el tubo orotraqueal y traqueotomía, catéter venoso central y la sonda vesical de demora. Hubo correlación fuerte y significativa entre el resultado óbito y la cantidad de infecciones presentes y al número de patógenos aislados en cada paciente.

En relación con el análisis realizado en este estudio, es importante que se realicen otras investigaciones por un periodo de tiempo y muestras mayores, a fin de obtenerse resultados más concretos y que puedan dar sustento a la práctica asistencial, pues las IRAS son responsables por excesiva carga del cuidado crítico, impactando negativamente en el presupuesto de estado, contribuyendo a una reducción de inversiones en otras áreas, como la atención básica, además de aumentar las tasas de mortalidad en estos ambientes.

Es imprescindible la correcta investigación y vigilancia de los casos de IRAS, atendiendo los diferentes factores asociados a esa condición, tales como: susceptibilidad aumentada del paciente, aparición de bacterias multirresistentes debido al uso inadecuado e indiscriminado de antibióticos, falta de trabajo sistematizado por parte del equipo de salud y procedimientos asistenciales sin la debida técnica aséptica. Se trata pues, de un asunto de extrema complejidad, siendo necesario un gran esfuerzo para la resolución de tal problemática, resultando evidente la necesidad de vigilancia epidemiológica de las IRAS en pacientes internados en la Unidad de Cuidados Intensivos.

El control de las IRAS es un trabajo laborioso que exige cada vez más un esfuerzo y trabajo multiprofesional dentro de la $\mathrm{UCl}$, a fin de minimizar los impactos traídos por estas complicaciones, volviendo el servicio más eficiente y eficaz. Tales acciones pueden concentrarse en el fortalecimiento de las acciones de educación en salud de los profesionales para la prevención de las IRAS, higienización de las manos en todos 
los momentos de la asistencia, control rigoroso de los procedimientos en el ambiente crítico, implementación de bundles para la prevención de los principales tipos de infección, monitoreo y manejo terapéutico adecuado de los casos de IRAS, divulgación de datos epidemiológicos al equipo, incentivo para el uso de equipamientos de protección individual, entre otras.

En este sentido, es imprescindible el fortalecimiento de la $\mathrm{CCHI}$ de la institución investigada, para que pueda actuar como balizadora y articuladora de las acciones y servicios que van al encuentro del problema, con el fin de cualificar el cuidado al paciente crítico.

Entre las limitaciones de la investigación, se destaca el periodo relativamente corto de los casos analizados y el número de casos evaluados en el estudio que no permite la generalización de los hallazgos para la población en general.

\section{REFERENCIAS}

1. Silva PF, Padoveze MC. Infecções relacionadas a serviços de saúde orientação para público em geral: Conhecendo um pouco mais sobre infecção. 2012. Disponível em: http://pesquisa.bvs.br/brasil/resource/pt/ses-27259.

2. Freire ILS, Menezes LCC, Sousa NML, Araújo RO, Vasconcelos QLDAQ, Torres GV. Epidemiologia das infecções relacionadas à assistência à saúde em unidade de terapia intensiva pediátrica. Revista de Atenção à Saúde, Rio Grande do Norte-RN. no 35, jan./mar. 2013. Disponível em: http://seer.uscs.edu.br/index.php/revista ciencias saude/article/view/1675/1371

3. Viana RAPP, Whitaker IY. Enfermagem em terapia intensiva: Práticas e Vivências. Porto Alegre-PR: Artmed, 2011.

4. Brasil. Agência Nacional de Vigilância Sanitária. Medidas de Prevenção de Infecção Relacionada à Assistência à Saúde Brasília, DF, 1a edição, 2013b. Disponível em: http://www20.anvisa.gov.br/segurancadopaciente/images/documentos/livros/Livro4-

MedidasPrevencaolRASaude.pdf.

5. Calil K, Valente GSC, Silvino ZR. Ações e/ou intervenções de enfermagem para prevenção de infecções hospitalares em pacientes gravemente enfermos: Uma Revisão Integrativa. Enfermería Global, Murcia-Espanha, v. 13, n. 2, p. 425-443, abr. 2014. Disponível em: http://revistas.um.es/eglobal/article/view/156491.

6. Nogueira PSF, Moura ERF, Costa MMF, Monteiro WMS, Brondi L. Perfil da infecção hospitalar em um hospital universitário. Revista de Enfermagem UERJ. Rio de Janeiro-RJ, v. 17, n. 1, p. 96-101, jan./mar. 2009. Disponível em: http://www.facenf.ueri.br/v17n1/v17n1a18.pdf.

7. Oliveira AC, Kovner CT, Silva RS. Infecção hospitalar em unidade de tratamento intensivo de um hospital universitário brasileiro. Rev. Latino-Americana Enfermagem, Ribeirão Preto-SP, v.18, n. 2, p. 97-104, mar./abr. 2010. Disponível em: http://www.scielo.br/pdf/rlae/v18n2/pt 14.pdf.

8. Oliveira AC, Paula AO, lquiapaza RA, Lacerda ACS. Infecções relacionadas à assistência em saúde e gravidade clínica em uma unidade de terapia intensiva. Revista Gaúcha de Enfermagem, v. 33, n. 3, p. 89-96, 2012. Disponível em: http://seer.ufrgs.br/index.php/RevistaGauchadeEnfermagem/article/view/25068/21950. 9. Nangino GO, Oliveira CD, Correia PC, Machado NM, Dias ATB. Impacto financeiro das infecções nosocomiais em unidades de terapia intensiva em hospital filantrópico de Minas Gerais. Rev. Bras. Ter. Intensiva, v. 24, n. 4, p. 357-361, 2012. Disponível em: http://www.scielo.br/pdf/rbti/v24n4/a11v24n4.pdf. 
10. Gomes AC, Carvalho PO, Lima ETA, Gomes ET, Valença MP, Cavalcanti ATA. Caracterização das infecções relacionadas à assistência à saúde em unidade de terapia intensiva. Revista de Enfermagem UFPE, v. 8, n. 6, p. 1577-1585, 2014. Disponível

em: http://www.revista.ufpe.br/revistaenfermagem/index.php/revista/article/view/5618.

11. Avci M, Ozgenc O, Coskuner SA, Olut Al.Hospital acquired infections (HAI) in the elderly: comparison with the younger patients. Archives of gerontology and geriatrics, v. 54, n. 1, 2012. Disponível em: http://www.aggjournal.com/article/S01674943(11)00082-3/pdf.

12. Pereira FGF, Chagas ANS, Freitas MMC, Barros LM, Caetano JÁ. Caracterização das infecções relacionadas à assistência à saúde em uma Unidade de Terapia Intensiva. Vigilância Sanitária em Debate: Sociedade, Ciência \& Tecnologia, v. 4, n. 1, p. 70-77, 2016. Disponível em: https://visaemdebate.incqs.fiocruz.br/index.php/visaemdebate/article/view/614.

13. Lisboa T, Nagel F. Infecção por patógenos multi-resistentes na UTI: como escapar. Rev Bras Ter Intensiva, v. 23, n. 2, p. 120-4, 2011. Disponível em: http://www.scielo.br/pdf/rbti/v23n2/a03v23n2.pdf.

14. Marra AR, Aranha $\underline{\mathrm{CLF}}$, Campos $\underline{\mathrm{PAC}}$, Teresa $\underline{\mathrm{S}}$, Petersen BPR. Nosocomial bloodstream infections in Brazilian hospitals: analysis of 2,563 cases from a prospective nationwide surveillance study. Journal of clinical microbiology, v. 49, n. 5, p. 1866-1871, 2011. Disponível em: http://scicurve.com/paper/21411591.

15. Costa FM, Soares AP, Batista LB, Carneiro JÁ, Santos JAD. Infecção hospitalar: distribuição topográfica e microbiológica em um hospital público de ensino. J Health Sci Inst, v. 32, n. 3, p. 265-70, 2014. Disponível em: https://www.unip.br/comunicacao/publicacoes/ics/edicoes/2014/03 jul-

set/V32 n3 2014 p265a270.pdf.

16. Favarin SS, Camponogara S. Perfil dos pacientes internados na unidade de terapia intensiva adulto de um hospital universitário. Revista de Enfermagem da UFSM, v. 2, n. 2, p. 320-329, 2012. Disponível em: https://periodicos.ufsm.br/reufsm/article/view/5178.

17. Brasil. Agência Nacional de Vigilância Sanitária. Critérios Diagnósticos de Infecções Relacionadas à Assistência à Saúde. Brasília, Distrito Federal, 1a edição, 2013a. Disponível em: http://www20.anvisa.gov.br/segurancadopaciente/images/documentos/livros/Livro2CriteriosDiagnosticosIRASaude.pdf.

18. Rodrigues PMA, Neto EC, Santos LRC, Knibel MF. Pneumonia associada à ventilação mecânica: epidemiologia e impacto na evolução clínica de pacientes em uma unidade de terapia intensiva. Jornal Brasileiro de Pneumologia, v. 35, n. 11, p. 1084-1091, $2009 . \quad$ Disponível em: http://www.scielo.br/scielo.php?script=sci arttext\&pid=S1806-37132009001100005.

19. Barros LM, Bento JNC, Caetano JÁ, Moreira RAN, Pereira FGF, Frota NM, et al. Prevalência de micro-organismo e sensibilidade antimicrobiana de infecções hospitalares em unidade de terapia intensiva de hospital público no Brasil. Revista de Ciências Farmacêuticas Básica e Aplicada, v. 33, n. 3, p. 429-435, 2012. Disponível em:

bib.fcfar.unesp.br/seer/index.php/Cien Farm/article/viewFile/2211/1267.

20. Brasil. Ministério da Saúde. Portaria n. 2.616, 12 de maio de 1998. Diário Oficial, Brasília, $1998 . \quad$ Disponível em: http://bvsms.saude.gov.br/bvs/saudelegis/gm/1998/prt2616 1205 1998.html.

21. Veras AS, Silva MRP, Carvalho MM, Carvalho LRB, Landim CAP. Perfil das infecções hospitalares nas unidades de terapia intensiva de um hospital de urgência. 
Revista de Enfermagem UFPE, v. 10, supl. 1, p. 194-201, 2016. Disponível em: https://periodicos.ufpe.br/revistas/revistaenfermagem/article/view/10940.

22. Fraimow HS, Tsigrelis C. Antimicrobial resistance in the intensive care unit: mechanisms, epidemiology, and management of specific resistant pathogens. Critical care clinics, v. 27, n. 1, p. 163-205, 2011. Disponível em: http://www.criticalcare.theclinics.com/article/S0749-0704(10)00099-0/pdf.

23. Souza ES, Belei RA, Carrilho CMDM, Matsuo T, Perugini MRE, Andrade G, et al. Mortalidade e riscos associados a infecção relacionada à assistência à saúde. Texto Contexto Enfermagem, v. 24, n. 1, p. 220-228, 2015. Disponível em: http://www.scielo.br/pdf/tce/v24n1/pt 0104-0707-tce-24-01-00220.pdf.

24. Hautemanière $A$, Florentin $A$, Hartemann $P$, Hunter PR. Identifying possible deaths associated with nosocomial infection in a hospital by data mining. American journal of infection control, v. 39, n. 2, p. 118-122, 2011. Disponível em: http://www.ajicjournal.org/article/S0196-6553(10)00665-6/pdf.

25. Vrijens F, Hulstaert F, Devriese S, Sande SV. Hospital-acquired infections in Belgian acute-care hospitals: an estimation of their global impact on mortality, length of stay and healthcare costs. Epidemiology and infection, v. 140, n. 01, p. 126-136, 2012. Disponível em: https://www.ncbi.nlm.nih.gov/pubmed/21320376.

26.Guimarães AC, Donalisio MR, Santiago THR, Freire JB. Óbitos associados à infecção hospitalar, ocorridos em um hospital geral de Sumaré-SP, Brasil. Rev Bras Enferm, v. 64, n. 5, p. 864-9, 2011. Disponível em: http://www.scielo.br/scielo.php?script=sci arttext\&pid=S0034-71672011000500010 